\title{
The usefulness of histopathological examinations of non-renal biopsies in the diagnosis of granulomatosis with polyangiitis
}

\author{
Anna Masiak ${ }^{1}$, Zbigniew Zdrojewski ${ }^{1}$, Rafał Pęksa ${ }^{2}$, Żaneta Smoleńska ${ }^{1}$, Zenobia Czuszyńska ${ }^{1}$, \\ Alicja Siemińska ${ }^{3}$, Bożena Kowalska ${ }^{4}$, Czesław Stankiewicz ${ }^{4}$, Bolesław Rutkowski ${ }^{5}$, \\ Barbara Butło-Piontecka ${ }^{5}$
}

${ }^{1}$ Department and Clinic of Internal Medicine, Connective Tissue Diseases and Geriatrics, Medical University of Gdansk, Poland

${ }^{2}$ Department of Pathomorphology, Medical University of Gdansk, Poland

${ }^{3}$ Department of Pulmonology and Allergology, Medical University of Gdansk, Poland

${ }^{4}$ Department and Clinic of Otolaryngology, Medical University of Gdansk, Poland

${ }^{5}$ Department and Clinic of Nephrology, Transplantology and Internal Diseases, Medical University of Gdansk, Poland

\begin{abstract}
Introduction: Granulomatosis with polyangiitis (GPA) is a rare, ANCA-associated, systemic disease characterized by necrotizing small and medium vessel vasculitis of unknown etiology associated with granulomatous inflammation affecting the renal, pulmonary, upper airways, ocular systems and other tissues. Histological proof of the granulomatosis with polyangiitis (GPA) can be obtained by biopsy of clinically involved sites. The main purpose of this study was to examine histopathological changes in non-renal biopsies from patients with established diagnosis of GPA and evaluated the histological confirmation at diagnosis of this disease.

Material and methods: A retrospective analysis was performed in patients with GPA diagnosed and treated in clinics of the University Clinical Center (UCK) in Gdansk in 1988-2009.

Results: In the analyzed group of GPA patients the histopathological examination of biopsies taken from involved tissues (except kidney) was performed in $60 \%$ of patients. Thirty-six out of 93 biopsies (39\%) were diagnosed as typical of GPA, 10 (10.7\%) were suggestive and 51 (54.8\%) were non-specific. Considering all biopsies, the diagnosis was confirmed in 24 patients ( $57 \%$ of patients in whom biopsies were taken). Epitheloid cell granulomas were present in 33 biopsies (43\%), characteristic necrosis in 27 biopsies (35\%), small vessel vasculitis in 18 biopsies (23\%), while multinucleated giant cells were identified only in 9 biopsies (12\%).

Conclusions: Histopathological examination of the affected tissues remains the gold standard of the diagnosis of GPA. Its usefulness increases, particularly in ANCA-negative patients, in the initial phase of the disease, or in patients with atypical clinical presentation. In many cases, it is necessary to repeat biopsy to establish the diagnosis. The role of the histopathological examination seems to be particularly important when ANCA is negative or clinical symptoms are atypical of GPA.
\end{abstract}

Key words: granulomatosis with polyangiitis, vasculitis, necrosis, histopathological findings.

\section{Introduction}

Granulomatosis with polyangiitis (GPA) is a rare, ANCA-associated, systemic disease characterized by necrotizing small and medium vessel vasculitis of unknown aetiology associated with granulomatous inflammation affecting the renal, pulmonary, upper airways, ocular systems and other tissues [1, 2]. Diagnosis of GPA is based on the definition of the disease established in the conference in Chapel Hill, United States of America in 1992 and on the criteria of classification developed in 1990 by 
the American College of Rheumatology (ACR) [1]. Nomenclature of vasculitides was revised in 2012 [2]. The 1990 American College of Rheumatology criteria for diagnosis of GPA include: (1) nasal or oral inflammation, (2) respiratory radiographic abnormalities consistent with respiratory tissue destruction (e.g., nodules, infiltrates, and cavities), (3) microhematuria or red blood cell casts on urinary sediment analysis, and (4) granulomatous inflammation on biopsy for pathology. Based on this classification, a diagnosis of GPA can be made with $88.2 \%$ sensitivity and $92.0 \%$ specificity when 2 out of the 4 criteria are met. The determination of serum ANCA can assist in the diagnosis of GPA, but ANCA positivity is not always conclusive for diagnosis, and the negativity for ANCAs is not sufficient to reject the diagnosis. Histopathological evidence of vasculitis remains the gold standard for diagnostic purposes [3]. The likely diagnostic yield varies and is dependent on the organ targeted. In histological terms, GPA consists of ischemic necrosis with a specific "geographical" organization, with the formation of a nonmicrobial neutrophilic abscess (microabscesses) and a polymorphic granuloma containing polymorphonuclear leukocytes, lymphocytes, plasma cells, dendritic cells, eosinophils and multinucleated giant cells. The vasculitis affects small-sized and middle-sized vessels, capillaries and venules [4, 5]. Adequate therapy requires an early diagnosis, but diagnosing ANCA-associated vasculitis (AAV) can be challenging. The typical necrotizing granulomas and vasculitis may be missed by the pathologists either in the early stage of the disease or in small fragments of tissue. Consequently, the diagnosis may be a challenge when the typical histological features are absent or when lungs and kidneys are not involved in disease or even the patient refers to an oncologically oriented or infectious department. The main purpose of this study was to examine histopathological changes in biopsies from patients with established diagnosis of GPA and evaluated the histological confirmation at diagnosis of this disease. The main focus has been put on biopsies other than kidney [6].

\section{Material and methods}

Seventy patients with GPA who were treated in single clinical hospital between 1988 and 2009 were included in this retrospective study. Before 1990 diagnosis was established according to specialist decision based on clinical presentation and histopathology. Since 1990 GPA was diagnosed in accordance with the classification criteria as mentioned above. Due to retrospective nature of this analysis, based on initial presentation of the disease, the patients were classified by authors to different forms of GPA defined by the European Vasculitis Study Group (EUVAS) [7]. The characteristic of the group is presented in Table I. Diagnostic evaluation included histo- pathological assessment of the collected tissue samples other than kidney biopsy. Each biopsy specimen result was evaluated as to the presence of granulomatous inflammation, multinucleated giant cell, necrosis and vasculitis, which are characteristic findings in GPA biopsies. Based on these four typical findings, the biopsies were classified according to Devaney [5] into three groups: typical of GPA (more than three findings), suggesting of GPA (two findings) and non-specific (less than two findings). The tissues were examined at the Department of Pathomorphology Medical University of Gdansk. ANCA antibodies were detected using an indirect immunofluorescence technique (IIF) and commercially available ELISA kit according to the manufacturer's instructions in Central Clinical Laboratory in Gdansk.

\section{Results}

Histopathological examination was conducted in $42(60 \%)$ subjects. Total number of 93 specimens of different tissues and organs were evaluated (except kidney) (Table II). In some cases, if necessary, biopsies were repeated

Table I. Characteristic of the analyzed group of GPA patients

\begin{tabular}{|c|c|}
\hline Number of patients & 70 \\
\hline Sex: female/male & $35 / 35$ \\
\hline $\begin{array}{l}\text { The mean follow-up time } \\
\text { (months) }\end{array}$ & $\begin{array}{l}80 \pm 60 \text { (median } 60) \\
\text { from } 6 \text { to } 216\end{array}$ \\
\hline Age at the diagnosis (years) & $47.4 \pm 14.5$ \\
\hline $\begin{array}{l}\text { The mean time from onset } \\
\text { of symptoms to diagnosis } \\
\text { (months) }\end{array}$ & $13.54 \pm 19.5$, from 1 to 96 \\
\hline Number of deaths & $22(31.4 \%)$ \\
\hline $\begin{array}{l}\text { Prevalent organ } \\
\text { involvement }\end{array}$ & $\begin{array}{c}\text { upper respiratory } \\
\text { tract }-82.8 \% \\
\text { kidneys }-58.5 \% \\
\text { lower respiratory tract - 57\% } \\
\text { skin }-38.5 \% \\
\text { nervous system - 37\% } \\
\text { gastrointestinal tract - 31.4\% } \\
\text { eye }-25.7 \% \\
\text { heart }-4.3 \% \\
\text { parotid glands }-4.3 \%\end{array}$ \\
\hline $\begin{array}{l}\text { The number of patients that } \\
\text { met the criteria } \\
\text { of the various forms } \\
\text { of GPA }\end{array}$ & $\begin{array}{l}\text { localized form }-10 \\
\text { early systemic form }-26 \\
\text { generalized form }-8 \\
\text { severe }-23 \\
\text { resistant }-3\end{array}$ \\
\hline $\begin{array}{l}\text { Antibodies (immunological } \\
\text { tests were performed in } \\
64 \text { patients) }\end{array}$ & $\begin{array}{c}\text { cANCA - } 58(83 \%) \\
\text { pANCA - } 1(1.5 \%) \\
\text { coincidence of pANCA } \\
\text { and cANCA - } 5(7 \%)\end{array}$ \\
\hline
\end{tabular}

CANCA - cytoplasmic anti-neutrophil cytoplasmic antibodies pANCA - perinuclear anti-neutrophil cytoplasmic antibodies 
Table II. Source of tissue for biopsy and their diagnostic usefulness in the confirmation of GPA diagnosis

\begin{tabular}{|lcccc|}
\hline Organ biopsy & $\begin{array}{c}\text { The total number } \\
\text { of collected tissue sections }\end{array}$ & $\begin{array}{c}\text { Findings typical } \\
\text { for GPA }\end{array}$ & $\begin{array}{c}\text { Findings } \\
\text { suggestive for GPA }\end{array}$ & $\begin{array}{c}\text { Nonspecific } \\
\text { findings }\end{array}$ \\
\hline Nasal mucosa & 25 & $8(32 \%)$ & $2(8 \%)$ & $15(60 \%)$ \\
\hline Larynx & 15 & $1(7 \%)$ & $2(13 \%)$ & $12(80 \%)$ \\
\hline Paranasal sinuses & 12 & $6(50 \%)$ & 0 & $6(50 \%)$ \\
\hline Fine-needle biopsy of lung & 8 & $4(80 \%)$ & 0 & $8(100 \%)$ \\
\hline Open lung biopsy and punch biopsy & 5 & $1(25 \%)$ & $3(75 \%)$ & $1(20 \%)$ \\
\hline Mucous membrane of the palate & 4 & $2(67 \%)$ & 0 & $1(33 \%)$ \\
\hline Bronchus & 3 & $1(33 \%)$ & 0 & $2(67 \%)$ \\
\hline The nasal part of the pharynx & 3 & $1(33 \%)$ & 0 & $2(67 \%)$ \\
\hline Skin & 3 & $2(67 \%)$ & $1(33 \%)$ & 0 \\
\hline Parotid gland & 3 & $1(50 \%)$ & 0 & $1(50 \%)$ \\
\hline Tongue & 2 & $1(100 \%)$ & 0 & 0 \\
\hline Intestine & 1 & 0 & 0 & $1(100 \%)$ \\
\hline Gingiva & 1 & $1(100 \%)$ & 0 & 0 \\
\hline Eyelid & 1 & 0 & $1(100 \%)$ & 0 \\
\hline The soft tissues of the face & 1 & $1(100 \%)$ & 0 & 0 \\
\hline The tissues of the orbit & 1 & $1(100 \%)$ & 0 & 0 \\
\hline The soft tissue of the abdominal wall & 1 & $1(33 \%)$ & $1(33 \%)$ & $1(33 \%)$ \\
\hline Ear & 1 & & 0 & 0 \\
\hline Post-mortem examination & 3 & & 0 & 0 \\
\hline
\end{tabular}

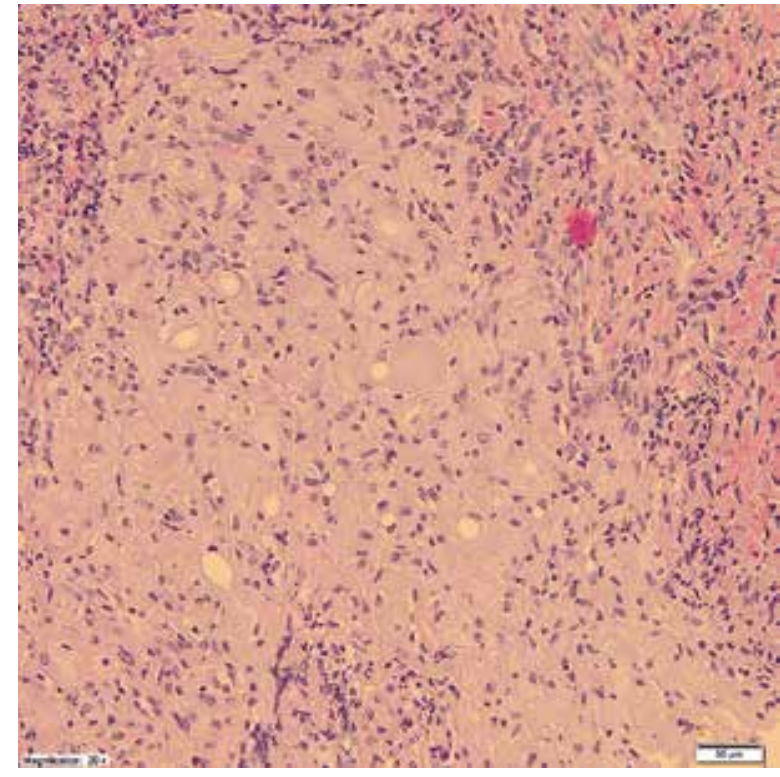

Fig. 1. Granuloma composed of histiocytes and surrounded mixed inflammatory cells including lymphocytes.

(during initial presentation of the disease or in relapses). The number of specimens collected from each patient ranged from 1 to 7 . The 36 out of 93 biopsies (39\%) were diagnosed as typical of GPA, 10 (10.7\%) were suggestive and 51 (54.8\%) were non-specific (Table II). Considering all bi-

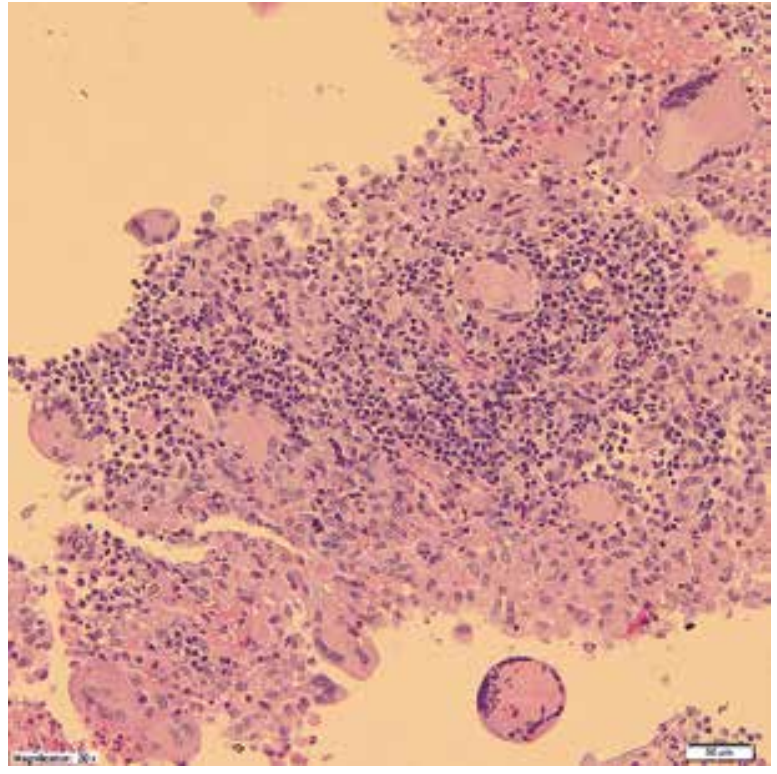

Fig. 2. Multinucleated giant cells and chronic inflammation.

opsies, the diagnosis was confirmed in 24 patients (57\% of patients in whom biopsies were taken). Typical findings in GPA biopsies consisted of granulomas (Fig. 1), multinucleated giant cell (Fig. 2), necrosis (Fig. 3) and vasculitis (Fig. 4). Seventeen histopathological descriptions contained only 


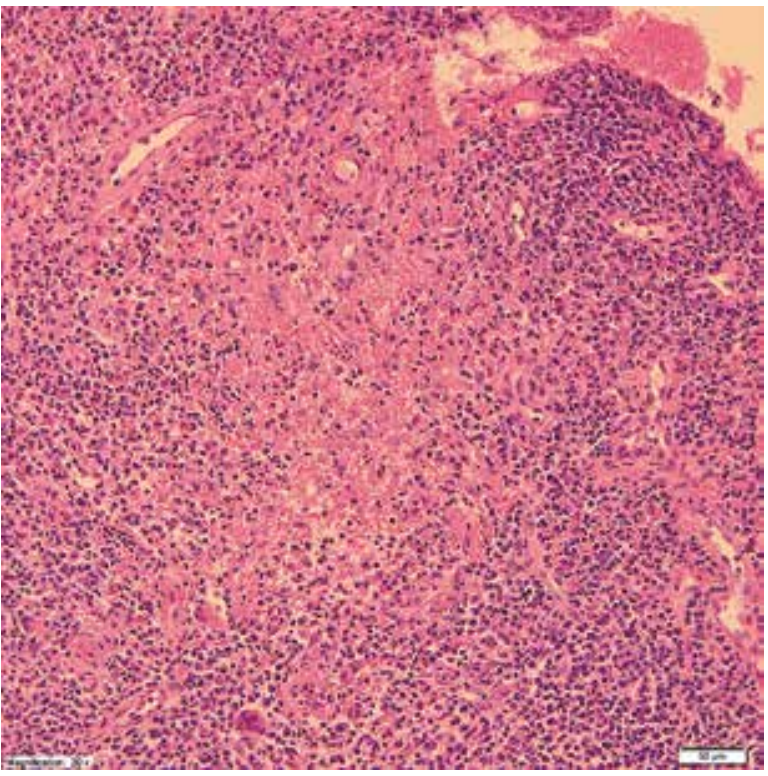

Fig. 3. Granulomatosis with polyangiitis has areas of necrosis that may be rimmed in part by multinucleated giant cells.

a summary without detailed description, so further analysis included 76 biopsies other than kidney. Necrosis was seen in 27 biopsies (35\%), small vessel vasculitis in 18 biopsies (23\%), epithelioid cell granulomas in 33 biopsies (43\%)

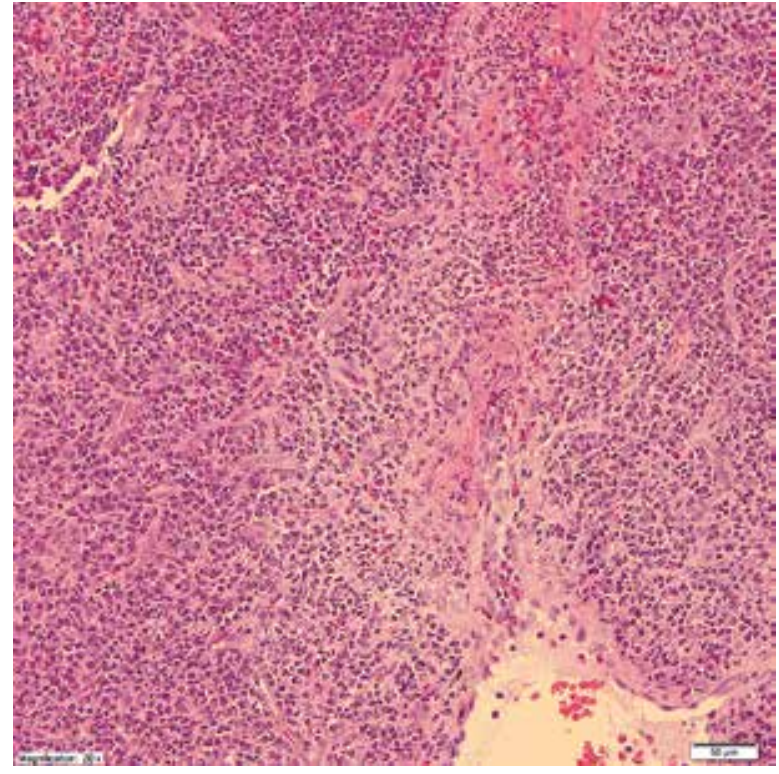

Fig. 4. Vasculitis of a small ortevy with adjacent granulomatosis inflammation.

while multinucleated giant cells were identified only in 9 biopsies (12\%) (Table III).

Table IV presents the histopathological findings in conjunction with the results of immunological tests.

Table III. Histologic changes in biopsy specimens in GPA

\begin{tabular}{|c|c|c|c|c|c|c|}
\hline \multirow[t]{2}{*}{ Site } & \multirow{2}{*}{$\begin{array}{l}\text { No. of samples } \\
\text { with complete } \\
\text { description }\end{array}$} & \multicolumn{5}{|c|}{ No. } \\
\hline & & necrosis & granulomas & giant cells & vasculitis & $\begin{array}{l}\text { non-specific } \\
\text { inflammation }\end{array}$ \\
\hline Nasal mucosa & 21 & 7 & 12 & 2 & 5 & 9 \\
\hline Larynx & 15 & 4 & 5 & 0 & 1 & 10 \\
\hline Paranasal sinuses & 9 & 3 & 6 & 1 & 1 & 6 \\
\hline Fine-needle biopsy of lung & 6 & 1 & 0 & 0 & 0 & 4 \\
\hline $\begin{array}{l}\text { Open lung biopsy and punch } \\
\text { biopsy }\end{array}$ & 4 & 3 & 2 & 2 & 1 & 1 \\
\hline Mucous membrane of the palate & 3 & 2 & 1 & 0 & 2 & 0 \\
\hline Bronchus & 3 & 2 & 1 & 1 & 1 & 1 \\
\hline The nasal part of the pharynx & 2 & 0 & 0 & 0 & 0 & 1 \\
\hline Skin & 3 & 1 & 0 & 0 & 3 & 0 \\
\hline Parotid gland & 2 & 1 & 1 & 1 & 0 & 1 \\
\hline Intestine & 1 & 1 & 0 & 0 & 1 & 0 \\
\hline Gingiva & 1 & 0 & 1 & 0 & 0 & 0 \\
\hline Eyelid & 1 & 0 & 1 & 1 & 0 & 0 \\
\hline $\begin{array}{l}\text { The soft tissue of the abdominal } \\
\text { wall }\end{array}$ & 1 & 0 & 1 & 0 & 0 & 0 \\
\hline Ear & 1 & 0 & 1 & 0 & 0 & 1 \\
\hline Post-mortem examination & 3 & 2 & 1 & 1 & 3 & 0 \\
\hline
\end{tabular}


Table IV. Results of histopathological examinations and ANCA test results in patients with GPA

\begin{tabular}{|lccc|}
\hline & \multicolumn{3}{c|}{ Histopathological examination } \\
\cline { 2 - 4 } & confirming & suggestive & non-specific \\
\hline ANCA $(+)$ & 30 & 2 & 15 \\
\hline ANCA $(-)$ & 5 & 1 & 2 \\
\hline ANCA no & 2 & 0 & 0 \\
\hline
\end{tabular}

Thirty patients with histopathologically confirmed GPA were found to be ANCA-positive. Fifteen patients without histological confirmation were ANCA-positive, while in only 5 patients with typical histopathological findings autoantibodies were not detected. It is necessary to mention that they were mainly patients with localized form of GPA. Two patients with non-specific histopathological findings and ANCA-negative were initially diagnosed as GPA based on a typical clinical feature. During further observation, both patients were found to be ANCA-positive during a relapse.

\section{Discussion}

Since the first description of granulomatosis with polyangiitis in 1931, our knowledge on its' pathogenesis, clinical picture and course has become much more profound. Gradual increase of the incidence of GPA is noted in recent years [8-10]. The cause of this phenomenon remains unknown, but this is surely partly due to the discovery and subsequent introduction of ANCA tests into common diagnostic evaluation [11].

In a patient suspected of having AAV (clinical features of GPA, MPA or EGPA and a positive test for ANCA), a biopsy should be performed to confirm the diagnosis [3]. A positive ANCA test strongly suggests the diagnosis of vasculitis, but both diagnostically false-positive and false-negative results may be seen. Histologic examination of tissue obtained by biopsy of an affected organ remains the most definitive method to establish a diagnosis. This is particularly important in patients with atypical course of the disease and/or ANCA-negative. Prompt diagnosis is important to permit initiation of therapy that may be life-saving and organ sparing. Moreover, correct prognosis in patients with renal involvement can only be assessed based on renal biopsy.

Granulomatosis with polyangiitis has three distinctive histopathological features: necrosis, granulomatous inflammation and vasculitis. The histopathology of GPA is varied, and it is important to remember that often not all the characteristic features are seen on a biopsy, especially in the head and neck area [5]. The vasculitis associated with GPA begins as a mixed acute and chronic inflammatory infiltrate that can progress to granulo- matous and necrotizing vasculitis. The vasculitis of GPA typically shows fibrinoid necrosis affecting the walls of small to medium-sized vessels (capillaries, venules, arterioles and arteries) occasionally accompanied by granulomatous inflammation within the vessel wall [12]. Thrombosis of the lumen with subsequent fibrosis is often present. Endothelial cells are the target of the initial injury in vasculitis of GPA which leads to swelling, necrosis and detachment of endothelial cells. Granulomatous vasculitis is very characteristic but not always present. Patients may present with intrapulmonary hemorrhage and haemoptysis, skin vasculitis or glomerulonephritis. Immunoglobulin and/or complement deposits are fairly or not detected in glomerular lesions or at other sites. The term 'pauci-immune' is used for this reason.

Extra-vascular lesions of GPA include mainly granulomas, which are defined as a focus of primarily mononuclear inflammatory cells, including neutrophils, macrophages, epitheloid cells and multinucleated giant cells, surrounded by CD4+ T-cells, CD8+ T-cells, and sometimes eosinophils, CD20+ B lymphocytes and plasmacells [12]. The $T$ and $B$ lymphocytes may be diffusely distributed or organized as follicle-like structures. It is worth noting that especially in the acute lesions of GPA, the predominant pattern of inflammation is not granulomatous, but purulent. Thus, the inflammation has the appearance of an abscess more than a granuloma. Granulomas may produce large, irregular, so-called geographic areas of necrosis, typically containing neutrophilic nuclear basophilic debris. The central area of necrosis is acellular or may occasionally contains polymorphonuclear leucocytes and epithelioid histiocytes which may get arranged around the necrotic foci. Granulomas may be in lungs, bronchi (sometimes leading to tracheal or bronchial stenosis) or in the upper respiratory tract. Often, the only feature in the acute inflammatory lesions that is reminiscent of granulomatous inflammation is the presence of scattered multi-nucleated giant cells. Giant cells, which are formed by the coalescence of monocytes and macrophages into multinucleated syncytia, may be a component of granulomas, but it is worth remembering that giant cells are neither sufficient nor required for a diagnosis of granulomatous inflammation [13]. Giant cells are variable in number and are typically "triangular" with a rim of peripheral nuclei. The mucosal lesions consist of foci of necrotizing inflammation and ulceration with occasional multinucleate histiocytic giant cells. Non-specific changes occur around the periphery of the more typical areas and can be misleading in small biopsies. Even though the typical pathological triad includes the presence of vasculitis, granuloma and necrosis, it is rare for biopsy specimens to present with all three of them $[14,15]$. 
Maguchi et al. [4] analyzed histopathological results of tissue samples collected from patients with conclusively diagnosed GPA. They concluded that the most frequent abnormality was necrosis (51\% of specimens), then vasculitis (31\%), granulomas of epitheloid cells (27\%) and, least commonly, the presence of giant multinucleated cells (20\% of all biopsies). Depending on the number of detected abnormalities, the results were classified as typical for GPA (> 3 abnormalities), suggestive of GPA (2 abnormalities) or non-specific (1 abnormality). Based on this classification, they found out that out of all assessed specimens 24\% were typical, $18 \%$ were suggestive of GPA and as much as 58\% were non-specific [4]. Other researchers obtained comparable results: $41 \%$ [16] to $60 \%$ [17]. In our group of patients $43 \%$ non-renal biopsies were diagnosed as typical of GPA, $12 \%$ were suggestive and $44 \%$ were non-specific. Considering all biopsies, the diagnosis was confirmed in $63 \%$ of patients in whom biopsies were taken. This data clearly shows how difficult it is to obtain a conclusive confirmation of GPA, which, to a great extent, also depends on the location and method of sample collection [18]. The small amount of tissue available in biopsy specimens especially from the head and neck makes it difficult or impossible to identify pathologic features of GPA. Identification vasculitis is difficult due to the paucity of blood vessels when the specimens are small.

In the analyzed group of patients, the highest sensitivity was found for open pulmonary biopsy (80\%). Other researchers assessed its sensitivity to be even as much as $90 \%[14,16,18,19]$. It is worth noting that a specimen collected during transbronchial biopsy is of much lower diagnostic value (only 10\%) [20] and in the group of our patients none of all 8 performed transbronchial biopsies of the lung allowed correct diagnosis. Due to the relative easiness and safety of specimen collection, samples for histopathological examinations are most commonly collected from the upper respiratory tract [14-16]. Similarly to other studies $[18,21]$, the same in our patients, over $50 \%$ of biopsies revealed only a non-specific inflammation with necrosis. In general, the sensitivity of the histopathological examination of samples collected from the nose or paranasal sinuses was estimated to be approximately $20-50 \%[15,19]$. This data becomes particularly essential in reference to patients with limited disease. The fact that in this group even more than 30\% of subjects were ANCA-negative [22] clearly shows how difficult it may be to correctly diagnose GPA when it is located in the upper respiratory tract. Our results show that diagnostically-useful tissues in the head and neck regions are obtained in a decreasing order of availability, first from the paranasal sinus, then nose and larynx. In many cases, it is necessary to repeat biopsy and make repeated attempts to establish the diagnosis. In the majority of our patients, biopsies of affected organs were repeated several times in order to confirm diagnosis.

A broad spectrum of diseases should be considered in the differential diagnosis. Major entities that must be distinguished pathologically from GPA include granulomatous infection, lymphomatoid granulomatosis, sarcoidosis and foreign body reactions or the other form of small vessel vasculitis. Clinical data are very helpful in many cases. Necrotizing granulomatous inflammation occurs in fungal and mycobacterial infections. In rare cases vasculitis may also occur in these infections. In the course of tuberculosis and histoplasmosis the granulomas are often present. Presence of classical sarcoid-like epitheloid and giant cell granulomas should alert the pathologist to other possible diagnoses, particularly tuberculosis. It's necessary to exclude infection by performing special stains or cultures for fungi or mycobacteria before making diagnosis of GPA. Granulomatous inflammation is seen most commonly in response to infection, but may also occur in response to unknown causes, for example in sarcoidosis, Crohn's disease or eosinophilic granulomatosis with polyangiitis [23].

Infectious processes giving rise to pus or granulomas must be differentiated from the microabscesses seen in GPA. Granulomatosis with polyangiitis may mimic IgG4-related disease (IgG4-RD) on histologic examination of some biopsies, especially those from head and neck sites [24]. IgG4 immunostaining is often performed in this context for differential diagnosis with IgG4-RD. One should be aware of the fact that IgG4+ cells can be remarkably increased in biopsies of GPA especially of the sinonasal and orbital/periorbital regions. Because the morphologic and clinical manifestations of GPA and IgG4-RD may overlap, it could be a significant diagnostic pitfall in the differential diagnosis of these 2 entities. Other histologic features, such as granulomas and necrotizing vasculitis, along with the serum ANCA, should be considered before making a diagnosis of IgG4-RD [24].

\section{Conclusions}

The diversity of clinical symptoms and lack of definitive diagnostic criteria make the diagnosis of GPA difficult. Patients require extensive laboratory and imaging diagnostic examinations, and most importantly, cooperation of physicians of different specialties. Histopathological examination of the affected tissues remains the gold standard of the diagnosis of GPA. Its usefulness increases, particularly in ANCA-negative patients, in the initial phase of the disease, or in patients with atypical clinical presentation. In many cases, it is necessary to repeat biopsy to establish the diagnosis. The role of the histopathological examination seems to be particularly 
important when ANCA is negative or clinical symptoms are atypical of GPA.

The authors declare no conflict of interest.

\section{References}

1. Leavitt RY, Fauci AS, Bloch DA, et al. The American College of Rheumatology 1990 criteria for the classification of Wegener's granulomatosis. Arthritis Rheum 1990; 33: 1101-1107.

2. Jennette JC, Falk RJ, Bacon PA, et al. 2012 Revised International Chapel Hill Consensus Conference Nomenclature of Vasculitides. Arthritis Rheum 2013; 65: 1-11.

3. Yates M, Watts RA, Bajema IM, et al. EULAR/ERA-EDTA recommendations for the management of ANCA-associated vasculitis. Ann Rheum Dis 2016; 75: 1583-1594.

4. Maguchi S, Fukuda S, Takizawa M. Histological findings in biopsies from patients with cytoplasmic-antineutrophil cytoplasmic antibody (cANCA)-positive Wegener's granulomatosis. Auris Nasus Larynx 2001; 28: S53-S58.

5. Devaney KO, Travis WD, Hoffman G, et al. Interpretation of head and neck biopsies in Wegener's granulomatosis: a pathologic study of 136 biopsies from 70 patients. Am J Surg Pathol 1990; 14: 555-564.

6. Aasarød K, Iversen BM, Hammerstrøm J, et al. Wegener's granulomatosis: clinical course in 108 patients with renal involvement. NDT 2000; 15: 611-618.

7. Mukhtyar C, Guillevin L, Cid MC, et al. EULAR Recommendations for management of primary small and medium vesse vasculitis 2009. Ann Rheum Dis 2009; 68: 310-317.

8. Watts RA, Lane SE, Bentham G, et al. Epidemiology of systemic vasculitis. A ten-year study in the United Kingdom. Arthritis Rheum 2000; 43: 414-419.

9. Koldingsnes W, Nossent H. Epidemiology of Wegener's granulomatosis in Northern Norway. Arthritis Rheum 2000; 43: 2481-2487.

10. Mohammad AJ, Jacobsson LT, Mahr AD, et al. Prevalence of Wegener's granulomatosis, microscopic polyangiitis, polyarteritis nodosa and Churg-Strauss syndrome within a defined population in southern Sweden. Rheumatology 2007; 46 1329-1337.

11. Van der Woude FJ, Rasmussen N, Lobatto S, et al. Autoantibodies against neutrophils and monocytes: tool for diagnosis and marker of disease activity in Wegener's granulomatosis. Lancet 1985; 1: 425-429.

12. Wiatr E, Gawryluk D. New aspects of the pathogenesis of Wegener's granulomatosis. Pneumon Alergol Pol 2002; 70: 326-333.

13. Jennette JC. Nomenclature and classification of vasculitis: lessons learned from granulomatosis with polyangiitis (Wegener's granulomatosis). Clin Exp Immunol 2011; 164 (Suppl 1): 7-10.

14. Hoffman GS, Kerr GS, Leavitt RY, et al. Wegener's granulomatosis: an analysis of 159 patients. Ann Intern Med 1992; 116: 488-498.

15. Jennings CR, Jones NS, Dugar J, et al. Wegener's granulomatosis - a review of diagnosis and treatment in 53 subjects. Rhinology 1998; 36: 188-191.

16. Anderson G, Coles ET, Crane M, et al. Wegener's granuloma. A series of 265 British cases seen between 1975 and 1985. A report by a sub-committee of the British Thoracic Society Research Committee. Q J M 1992; 83: 427-438.

17. Stone JH. The Wegener's Granulomatosis Etanercept Trial Research Group: Limited versus severe Wegener's granulomatosis. Baseline data on patients in the Wegener's granulomatosis Etanercept Trial. Arthritis Rheum 2003; 48: 2299-2309.

18. Lie JT. Biopsy diagnosis of systemic vasculitis. Baillieres Clin Rheumatol 1997; 11: 219-236.

19. Langford C. How can relapses be detected and prevented in primary systemic small-vessel vasculitides? Clin Rheumatol 2005; 19: 307-320.

20. Schnabel A, Holl-Ulrich K, Dalhoff K, et al. Efficacy of transbronchial biopsy in pulmonary vasculitides. Eur Respir J 1997; 10: 2738-2743.

21. Takwoingi YM, Dempster JH. Wegener's granulomatosis: an analysis of 33 patients seen over a 10-year period. Clin Otolaryngol 2003; 28: 187-194.

22. Bajema IM, Hagen EC, Weverling-Rijnsburger AW, et al. Wegener's granulomatosis: a meta-analysis of 349 literary case reports. J Lab Clin Med 1997; 129: 17-22.

23. Lamprecht P, Trabandt A, Gross W. Clinical and immunological aspects of Wegener's granulomatosis (WG) and other syndromes resembling WG. IMAJ 2000; 2: 621-626.

24. Chang SY, Keogh KA, Lewis JE, et al. IgG4-positive plasma cells in granulomatosis with polyangiitis (Wegener's): a clinicopathologic and immunohistochemical study on 43 granulomatosis with polyangiitis and 20 control cases. Human Pathol 2013; 44: 2432-2437. 